\title{
Designing an Animated Character System for American Sign Language
}

\author{
Danielle Bragg \\ University of Washington \\ Seattle, WA, USA \\ dkbragg@cs.washington.edu
}

\author{
Raja Kushalnagar \\ Gallaudet University \\ Washington, DC, USA \\ raja.kushalnagar@gallaudet.edu
}

\author{
Richard Ladner \\ University of Washington \\ Seattle, WA, USA \\ ladner@cs.washington.edu
}

\begin{abstract}
Sign languages lack a standard written form, preventing millions of Deaf people from accessing text in their primary language. A major barrier to adoption is difficulty learning a system which represents complex 3D movements with stationary symbols. In this work, we leverage the animation capabilities of modern screens to create the first animated character system prototype for sign language, producing text that combines iconic symbols and movement. Using animation to represent sign movements can increase resemblance to the live language, making the character system easier to learn. We explore this idea through the lens of American Sign Language (ASL), presenting 1) a pilot study underscoring the potential value of an animated ASL character system, 2) a structured approach for designing animations for an existing ASL character system, and 3) a design probe workshop with ASL users eliciting guidelines for the animated character system design.
\end{abstract}

\section{ACM Classification Keywords}

H.5.m. Information Interfaces and Presentation (e.g. HCI): Miscellaneous

\section{Author Keywords}

American Sign Language (ASL); Character Systems; Reading and Writing; Literacy; Animation; Deaf; Design; si5s.

\section{INTRODUCTION}

Like other sign languages, ASL is a movement-based language without a standard written form. ${ }^{1}$ Worldwide, there are about 70 million people using a sign language as their primary language [42], and in the US and most of Canada, ASL is the primary language of the Deaf community. ${ }^{2}$ Many hearing people use sign languages, too. ASL has become the third-most popular language in US higher education, behind only French and Spanish, and has increasing enrollment [39].

\footnotetext{
${ }^{1} \mathrm{ASL}$ is our focus, but much applies to sign languages generally.

${ }^{2}$ Capital Deaf refers to the culture, lowercase deaf to hearing status.

Permission to make digital or hard copies of all or part of this work for personal or classroom use is granted without fee provided that copies are not made or distributed for profit or commercial advantage and that copies bear this notice and the full citation on the first page. Copyrights for components of this work owned by others than the author(s) must be honored. Abstracting with credit is permitted. To copy otherwise, or republish, to post on servers or to redistribute to lists, requires prior specific permission and/or a fee. Request permissions from permissions@ acm.org.

ASSETS '18, October 22-24, 2018, Galway, Ireland

(C) 2018 Copyright held by the owner/author(s). Publication rights licensed to ACM. ISBN 978-1-4503-5650-3/18/10 . \$ $\$ 15.00$

DOI: https : //doi .org/10.1145/3234695. 3236338
}

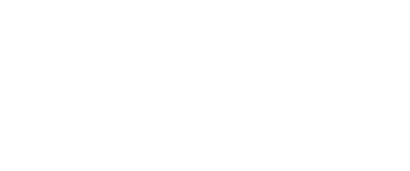

Figure 1: YOU GO TO SCHOOL TOMORROW. in a) si5s, and b) our animated si5s prototype. The movement symbols in a) (the arcs and dots) are replaced by animating the referenced handshape symbols to create b). (Note: This figure should be animated when viewed in Adobe Reader.)

Though ASL is not typically written, much of society and the technical world communicates through written text, excluding many ASL users from equal participation. A standard written form of ASL would make text-based platforms newly usable in ASL, including email clients, text messengers, social media, text editors, and much of the internet, as well as text-based resources like books and newspapers. It would also satisfy many Deaf people's desire to express themselves through writing in their primary language (e.g., [56]). Other possible benefits include promotion and dissemination of the language, and low-cost documentation and cultural preservation [22, 37].

Using English for reading and writing is insufficient. ASL is its own language, with a unique grammar and vocabulary; it is not signed English. Furthermore, many people are deaf from a young age, which can make learning English extremely difficult. Consequently, English literacy rates are low, with the average deaf high-school graduate reading at a fourth-grade level [27]. English is not the primary language of the Deaf community, and ultimately does not provide access to text in their primary language, unlike an ASL character system.

Historically, character systems worldwide have been defined by stationary (non-moving) features, but stationary characters do not intuitively represent sign language. Our paper medium, which only supports stationary displays, has limited character design in this way. Representing the 3D movements of ASL in stationary 2D notation is difficult. As a result, the extent to which a stationary character system can resemble live signing is limited, making it difficult to learn and use.

Unlike paper, digital displays support animation, providing an opportunity to create character systems that more closely resemble sign languages. We increasingly read on computerized devices, so characters no longer need to be limited to stationary designs. Incorporating animation in text is particularly relevant to sign languages like ASL, which are movement-based. Notably, unlike stationary symbols, animation can easily show gradation in sign speed, which has semantic meaning in ASL. 
In this work, we create the first animated sign language character system prototype. Like Chinese characters and heiroglyphic logograms, our animated characters represent individual signs, and form lines of text (e.g., Figure 1). Each character is composed of a configuration of symbols, some of which may be moving. These characters are based on si5s [3], formed by replacing si5s movement symbols with actual movement on the screen. Using animation allows us to create text that visually resembles sign movements. This visual resemblance to the live language has the potential to make character systems easier to learn and lower the barrier to adoption. Key contributions of this work are:

- An animated character system prototype for ASL, derived from an existing stationary character system, si5s [3].

- A survey and pilot study pointing to the need for an animated character system for ASL.

- Identification of design dimensions for representing sign movements in a character system, and guidelines for appropriate designs based on a workshop with ASL users.

\section{RELATED WORK}

Related work spans ASL linguistic work decomposing ASL into features that character systems represent, existing ASL character systems whose iconicity (resemblance to the live language) is limited by their stationary symbols, ASL video which uses dynamic displays to closely resemble live ASL, the emergence of animated text, and participatory design.

\section{ASL Linguistics}

ASL is a natural language, with fundamental linguistic components including phonology, morphology, syntax, and semantics [58]. Stokoe performed the initial linguistic analysis that helped establish ASL as a language, decomposing it into five types of features - handshape, location, orientation, movement, and relative position [50]. Features unrelated to movement can be represented in stationary character systems like si5s [3] (upon which our system is based) through fairly iconic symbols (e.g., a set of handshape symbols resembling various handshapes). Sign movements, often nuanced and complex, are more difficult to represent iconically in drawn symbols. By incorporating movement in characters, this work makes it possible to represent movement features much more iconically.

\section{ASL Writing and Character Systems}

There are two main types of ASL writing systems: 1) character systems, which present a set of symbols used according to a set of rules to represent the language, and 2) transliterations, which use another language's words but preserve ASL grammar. ASL transliterations are commonly provided through English gloss, which represents signs with English words in all-caps (e.g., BOOK), with additional symbols providing details. Gloss is popular for teaching ASL to hearing students who already know English. The rest of this section focuses on ASL character systems, which is the focus of our work.

ASL character systems for linguistics and computation are typically extremely detailed, which can make them difficult to learn and use. Stokoe notation is ASL's seminal linguistic notation system [51]. Subsequent linguistic notation systems are largely based on Stokoe's (e.g., [9, 32]), and are used by linguists to document and analyze language. Systems for computer modeling are often based on linguistic systems (e.g., HamNoSys [25]). Applications span digital transcription of linguistic corpora (e.g., iLex [24]), internal representation of signs for signing avatars (e.g., SigML [17]), and general motion transcription (e.g., [34, 36, 21, 26]).

ASL character systems for everyday use typically represent high-level information. Their symbols often visually resemble sign components (e.g., SignWriting [55], si5s [3], SignFont [41], ASL-phabet [54], ASL Orthography, SLIPA [43], and ASLSJ [52]). The 2D line-drawings used to represent complex 3D movements can be difficult to learn and use. By incorporating movement into text, we introduce the possibility of representing sign movements iconically in text, thereby reducing these difficulties. Our animated character system builds off of si5s, for reasons explained in the design section.

Growing technical support for ASL character systems underscores demand for digital ASL text. Compatibility with keyboards (e.g., [45]) and SMS (e.g., [1]) has been explored. Unicode characters reserved for SignWriting [11] provide cross-platform support, and markup languages like SWML support non-sequential scripts like SignWriting [12]. This work provides further evidence of demand, and further enhances digital ASL text by introducing animation to ASL character design.

\section{ASL Video}

ASL video content comprises 1) recordings of people signing and 2) animated avatars programmed to execute signs. Systems with live signers include real-time video calls, video relay services (VRS), and persistent resources such as websites and educational tools (e.g., [4, 16, 54, 53]). Avatar systems pass low-level sign representation (see above) through graphics engines (e.g., [29, 60]). Avatar generation is an open research area, often attempted through translation from English (e.g., $[62,28,40])$. Replacing text with video can introduce layout problems, potentially addressed through videos layered on existing content [15], or tooltips with videos or pictures [44]. Work on generating motion from notation and avatar sign speeds (e.g., [30]) can inform human-like movements for text. However, application to text requires foundational studies on animated text perception.

While video is a natural, powerful medium for ASL [31], it does not provide the functionality of text. Unlike text, video presents content through a specific body rather than abstractly, does not easily integrate into text-based platforms, and does not readily meet other text-met needs, e.g., discrete note-taking and iterative, collaborative content generation. An animated character set would provide the functionality of text, with the dynamic expressiveness of video. We do not seek to replace ASL video, rather to create a more iconic form of ASL text.

\section{Animated Text}

Animation is increasingly being integrated into text. Kinetic typography [10] originally introduced animation to text in film and advertising, using animation for special effects. Livefonts 
[7], alternate scripts that use animation to differentiate letters, provide a precedent for using animation as a defining feature of a writing system. Livefonts are a subset of Smartfonts [6], alternate scripts conceptually similar to Braille, that leverage the capabilities of modern screens (color, shape, and animation) to enhance the reading experience. Animated emoji have also been integrated into many text-based platforms including Skype and Facebook to enhance communication, but generally lack a vocabulary of ASL signs. Animating ASL characters similarly leverages dynamic displays to enhance ASL text.

The psychophysics of reading animated text is an open research area. Researchers often use rapid serial visual presentation (RSVP) [19] to control reading speed, by displaying a single word on a screen and changing the display at the desired rate. Such dynamic text displays can increase reading speed [47]. Animation can also impact the emotion conveyed by text [59], and legibility [38]. There is also evidence that people can learn to read Livefonts [7]. While this past work has examined character systems for spoken languages, this work contributes an initial exploration of the effect of animating a character system for sign language, which is movement-based.

\section{Participatory Design}

Participatory design is a methodology for involving all stakeholders, meaning everybody effected by a technology (or other product), in the design process. Participatory design (originally co-operative design) empowers users by giving them a voice in the design process $[18,5]$. Methods include interviews and observations, low- and high- fidelity prototyping, design sessions, structured brainstorming, and workshops (as described in [49]), and more recently distributed methods to involve remote users online (e.g., [23, 13]).

Participatory design has been used in a range of fields beyond technology, and has a rich literature spanning politics, participation, and methodology (as described in [33]). In particular, people with disabilities have been successfully involved in designing accessible solutions through participatory design (e.g., people with dementia [35], children with special needs [20], and Deaf students [61]). We used participatory design principles to involve ASL users in our design probe workshop.

\section{OPPORTUNITY EVALUATION STUDY}

To better understand potential animated ASL character system users, we ran an online study involving three parts: 1) a survey on ASL character systems, 2) a pilot study on whether animation can make a character system easier to understand without training, and 3) feedback on animated vs. stationary character systems. It was designed to answer four main questions:

Q1. Do ASL community members want to read content in an ASL character system?

Q2. If so, what is preventing them from using ASL character systems?

Q3. Can animation make an ASL character system easier to understand without training?

Q4. Does the community of ASL users see value in creating an animated ASL character system?
Guess the meaning of the sign.

(Click the English answer choices to view the signs they represent.)
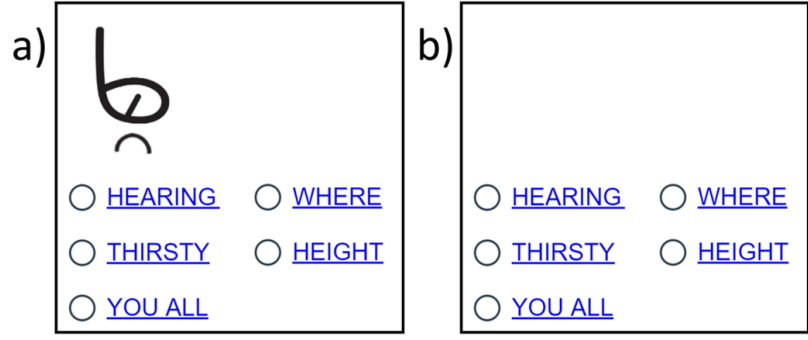

Figure 2: Sample identification questions for the sign WHERE, from text in a) si5s and b) animated si5s. (Viewed in Adobe Reader, b) contains animation.)

\section{Procedure}

The study was run online as a web study, and took 10-15 minutes total. After obtaining consent and asking basic demographic questions, the study consisted of three main parts:

Part 1: Survey questions on how people communicate in ASL on traditionally text-based platforms, and their usage of ASL character systems. The questions were multiple-choice, and allowed multiple selections and write-in options as needed.

Part 2: Pilot study on animated ASL character identifiability, probing whether animation can improve notation understandability without training. Participants were asked to identify signs from notation without training (Figure 2). Participants viewed notation for each sign in both animated and stationary forms, in order of increasing complexity. We randomized whether participants saw the animated or stationary set first. After viewing each set, participants provided qualitative feedback on how easy the signs were to identify, how enjoyable viewing them was, and how similar they looked to live signs.

Part 3: Feedback on the usefulness of animated ASL characters. We explicitly asked participants if they thought animating ASL characters can be valuable, and why (or why not).

\section{Identification Questions}

We based the pilot study (Part 2) on si5s because it naturally integrates animation (see Animation Design section for details). The stationary notation of each sign was presented in si5s. The animated version was created by replacing si5s movement symbols with animations. Specifically, the handshape symbols were animated according to the drawn trajectory, direction, or pattern. The animations were designed by the researchers based on experience with typography, animation, and ASL. The animations were created in PowerPoint, presented as GIFs, and played continuously.

The identification questions involved four signs: WHERE $^{3}$, UNDERSTAND $^{4}$, MAYBE $^{5}$, and MOTIVATION ${ }^{6}$, selected to span diverse movements (covering all si5s movement notation classes) and complexity (e.g., 1- vs. 2-handed). Each

\footnotetext{
${ }^{3}$ https: //Www . signingsavvy . com/sign/WHERE/478/1

${ }^{4}$ https://Ww . signingsavvy . com/sign/UNDERSTAND/715/1

$5_{\text {https: //WWW . signingsavvy . com/sign/MAYBE/261/1 }}$

$6_{\text {https : //WWW . signingsavvy . com/sign/MOTIVATION/3924/1 }}$
} 


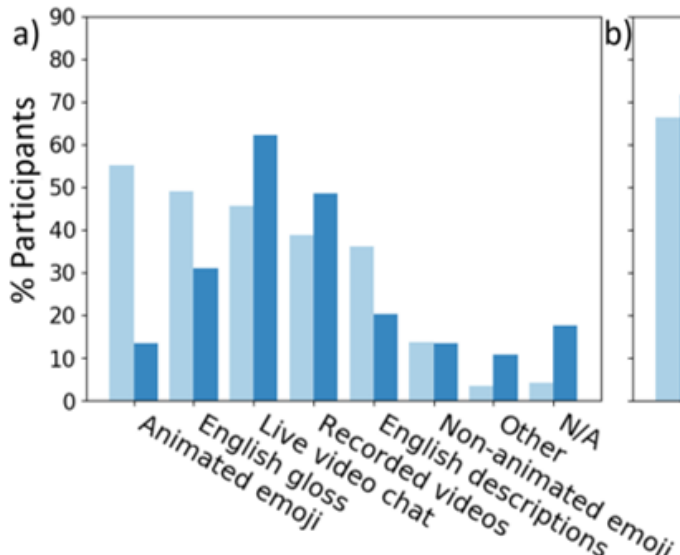

Digital ASL Communication Format

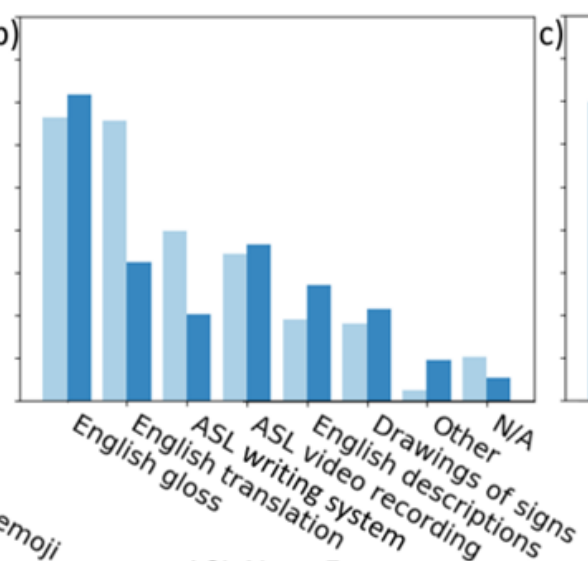

ASL Note Format

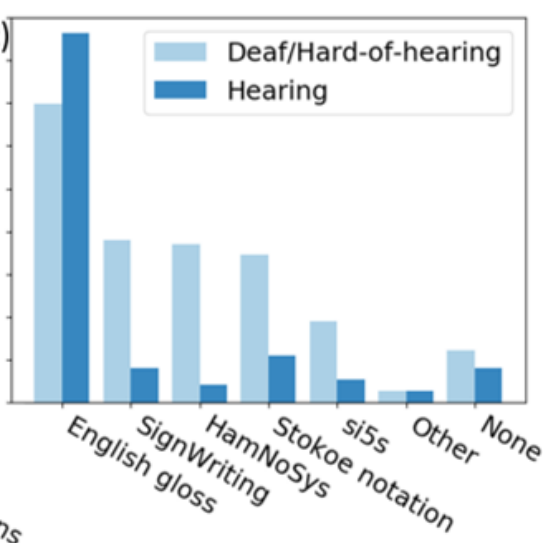

Known ASL Writing Systems

Figure 3: How people communicate in ASL a) digitally, b) when taking notes, and c) when using an ASL writing system.

question provided five multiple-choice answer options resembling the sign in question, ordered randomly. We selected featurally similar answer choices through Latent Semantic Analysis over a dataset of crowdsourced feature evaluations [8]. Each multiple-choice option was represented as an English word, with a link to a signed video from SigningSavvy [48], an online English-to-ASL dictionary.

\section{Participants}

ASL users were recruited online, through relevant email lists and social media, with IRB approval. In total, 195 participants completed the survey (74\% completion rate). ASL proficiency was self-reported, along the 6-point IRL Scale [46]. Basic demographics: Age: 9-77 $(\mathrm{m}=35)$; Gender: 147 (75\%) Female, 47 Male, 1 Other; Identity: 98 (50\%) Deaf, 18 (9\%) Hard-ofHearing, 74 (38\%) Hearing, 5 (3\%) Other; ASL Level:

\begin{tabular}{|l|l|}
\hline Level & \# Participants \\
\hline 0 - No proficiency & 0 \\
1 - Elementary proficiency & $21(11 \%)$ \\
2 - Limited working proficiency & $19(10 \%)$ \\
3 - Professional working proficiency & $64(33 \%)$ \\
4 - Full professional proficiency & $46(24 \%)$ \\
5 - Native or bilingual proficiency & $45(23 \%)$ \\
\hline
\end{tabular}

\section{Results}

The results from our opportunity evaluation study show a desire for an easily understood ASL character system, interest in an animated character system, and potential for animation to make character systems more immediately understandable.

\section{Q1: Need for ASL Character Systems}

The vast majority of participants reported a need or desire for an ASL character system. When explicitly asked which materials they want available in ASL text (Figure 4), the vast majority $-86 \%$ Deaf/Hard-of-hearing (DHH), $71 \%$ hearing participants - selected at least one material type, indicating a widespread desire for access to text in ASL.

Participants' means of communicating in ASL (Figure 3) further support the potential value of an animated ASL character system. A high percentage reported using some form of digital ASL communication ( $>95 \%$ DHH, $82 \%$ hearing), suggesting a pervasive desire for digital ASL communication. Despite the availability of video, text-based methods were the most

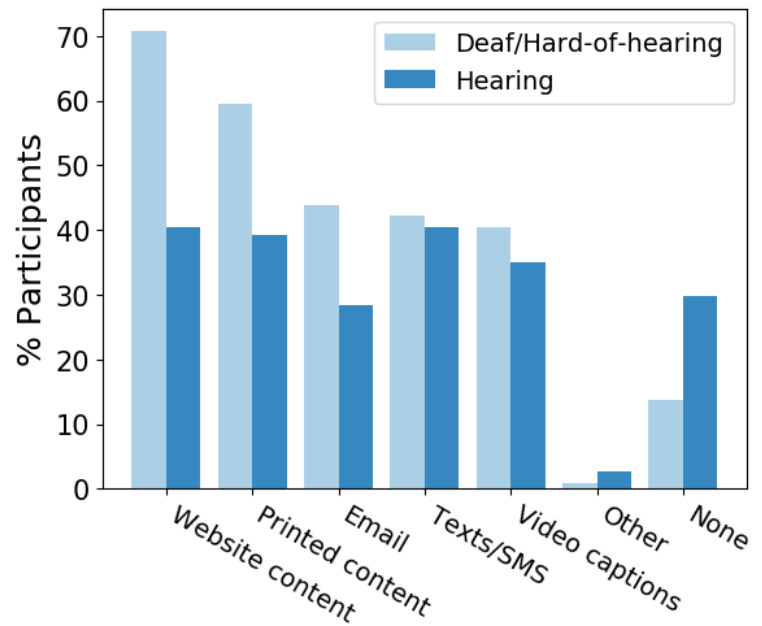

Material Desired in ASL Text

Figure 4: Materials participants wanted to read in ASL text.

common mediums among DHH (animated emoji, and English gloss), suggesting a need for ASL text, in addition to video. Animated emoji popularity (55\% DHH, 14\% hearing) suggests that integrating animation into text-based platforms is highly desirable, in particular for DHH people, which an animated character system would provide.

The vast majority of participants $(91 \%)$ reported taking notes for ASL content (Figure 3b), further supporting a need for reading/writing in ASL. Example use cases include preparing a vlog post, taking lecture notes, or writing a note or poem in ASL. The most-reported note-taking methods were text- (not video-) based, likely due to the ease, low cost, and inconspicuousness with which text can be created and edited.

Most participants ( $>90 \%)$ reported being able to read at least one ASL writing system (Figure 3c), indicating a wide need for them. English gloss was more popular among hearing participants, who are typically native English speakers using gloss to learn ASL. In contrast, ASL character systems were more popular among DHH than hearing participants, a difference underscoring the Deaf community's need for an ASL character system not based on English. We also note that our survey, and recruitment, was in English, excluding Deaf people who do not know English from participating, who we expect to be a 


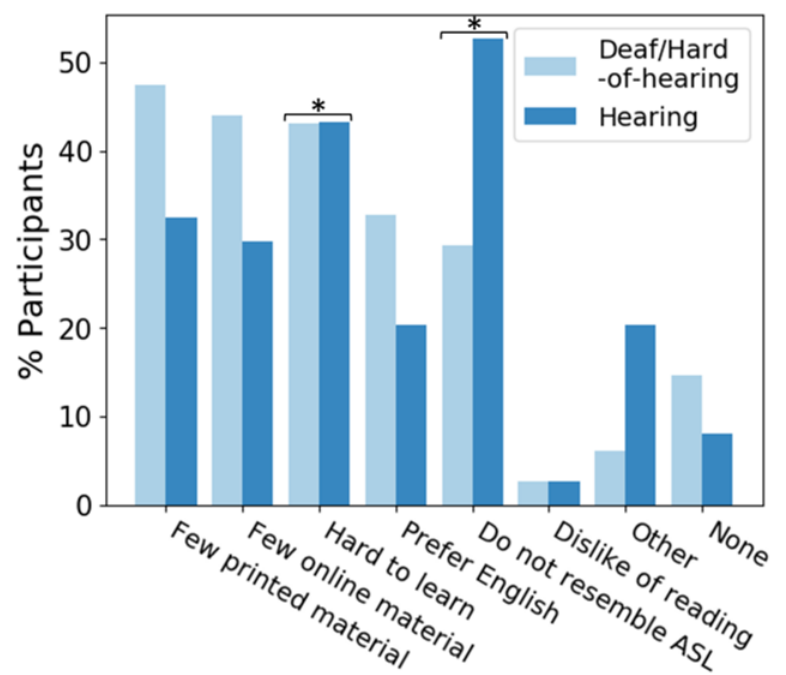

Barrier to Adoption

Figure 5: Barriers to using ASL character systems reported by participants. Barriers with asterisks are potentially addressed by introducing animation to character systems.

significant group based on prior literacy studies. Consequently, the fraction of DHH participants who report knowing English gloss is likely higher than for the DHH community at large.

\section{Q2: Unmet Needs with Existing ASL Character Systems}

Behind lack of materials, difficulty learning character systems was the most-reported adoption barrier for DHH participants, preventing adoption for $43 \%$ (Figure 5). Poor resemblance to ASL was the most common barrier for hearing participants, preventing adoption for $>50 \%$ of them. An animated character system is designed to address both these barriers.

An animated character system could also indirectly increase the availability of materials in an ASL character system. Character system adoption presents a circular startup problem. If people do not know the system, there is little incentive to produce materials; and if materials are not available, people are disincentivized to learn the system. Working towards a more learnable character system, we hope an animated character system can help break this cycle.

\section{Q3: Animated Character Understandability}

Figure 6 shows Identification Accuracy, the percent of participants who identified a sign from its notation correctly. Animated character identifiability was significantly higher for three of four signs (WHERE, UNDERSTAND, and MAYBE). ${ }^{7}$ We suspect that animating MOTIVATION did not improve identifiability due to poor design choices, as the animation speed more closely resembled the speed of other signs in the answer choices. The difference between groups who saw animated vs. stationary versions first was not significant $(p>.05)$, by a $\chi^{2}$ independence test for each question. Even for participants who reported familiarity with si5s, animation generally improved identifiability. These results suggest that animation can improve character identifiability, and highlight the importance of design choices in creating iconic animations.

\footnotetext{
${ }^{7}$ Note that the probability of randomly guessing correctly for a single question is .2. It follows that the probability of achieving $>=60 \%$ accuracy (e.g., animated character for WHERE) at random $<.000001$.
}

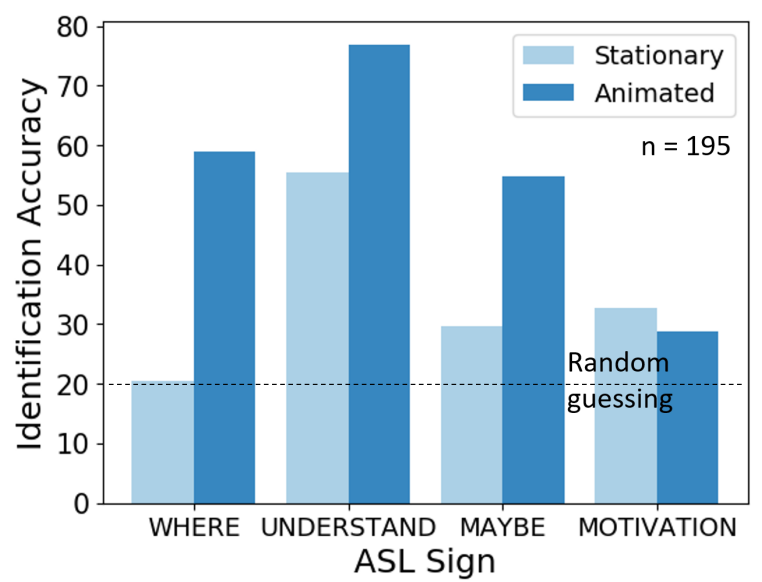

Figure 6: Identification Accuracy, the percent who identified signs from stationary vs. animated characters without training.

Participants took significantly less time to identify signs from the animated notation, suggesting that the animated versions were typically easier to recognize. Even participants who viewed the animated notation first were typically faster at identifying animated characters than stationary ones. The difference in identification times for animated vs. stationary versions was statistically significant $(p<.05)$ for each sign, except WHERE ( $p=.16)$, by t-tests.

\section{Q4: Qualitative Feedback}

Our animated characters received higher ratings than the stationary ones in terms of 1) similarity to live ASL, 2) ease of identification, and 3) viewing enjoyment. Figure 7 shows participants' assessments of similarity, ease, and enjoyment on a 7-point Likert scale. For the stationary characters, over $50 \%$ of participants reported negative assessment along the three dimensions, compared to under $40 \%$ for the animated versions. Overall, the distribution for each answer is skewed towards the positive for the animated characters, suggesting that animation can improve character set resemblance to live ASL, lower the learning barrier, and increase enjoyment.

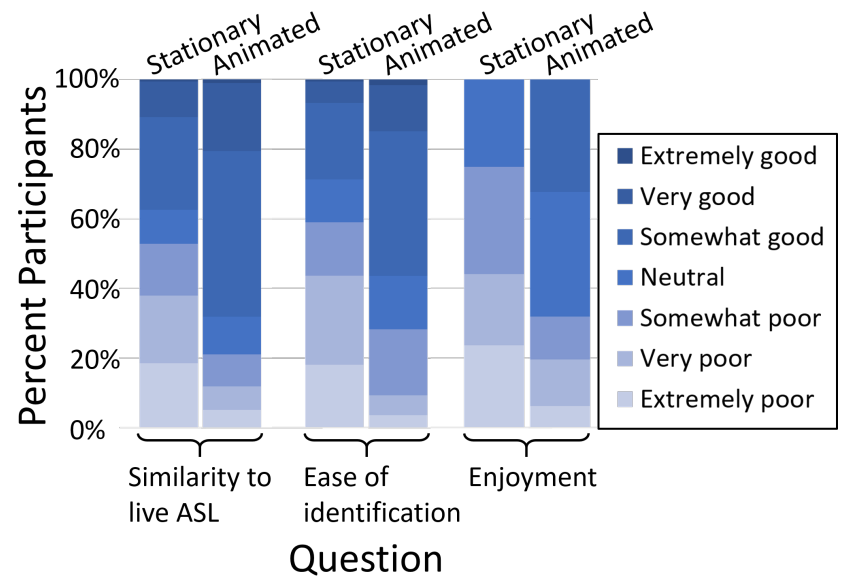

Figure 7: Participants' feedback on the animated vs. stationary characters in terms of similarity to live ASL, ease of identification, and enjoyment. Darker means better.

The vast majority of participants found value in animating ASL characters, as shown in Table 1. When asked at the end 
of the study, "Do you think that animating ASL characters can be valuable?", $71 \%$ responded Yes, $22 \%$ I'm not sure, and only $7 \%$ No. The overwhelmingly positive response to this question strongly suggests that animated ASL characters have potential value to the community, and merit further exploration.

\begin{tabular}{|l|r|r|}
\hline Answer & \% Participants & \# Participants \\
\hline Yes & $71 \%$ & 139 \\
I'm not sure & $22 \%$ & 43 \\
No & $7 \%$ & 13 \\
\hline
\end{tabular}

Table 1: Responses to "Do you think that animating ASL characters can be valuable?"

When asked "Why or why not?" after "Do you think that animating ASL characters can be valuable?" the 13 participants who answered "No" rejected ASL writing systems in general, not animation specifically. Some thought ASL is not suited to being written, explaining "ASL is not a written language and trying to fit it into that niche feels wrong." and "This is not what ASL is all about - it should be video based only." Others thought that learning a writing system was too hard, one participant explaining, "Too much work... Easier to type in English", and another "It is like learning a new ABC. We have to learn what each symbol means."

Participants who answered "No" or "I'm not sure" skewed towards high ASL proficiency and older, with proportional hearing statuses to the overall study population. They reported less desire and usage of ASL text, and higher adoption barriers. Their top barriers were poor iconicity and learnability, and their elevated accuracy in identifying animated characters suggests that animation could help address their main barriers.

\section{ANIMATION DESIGN}

How to design effective animations for a sign language character system is an open question that this work introduces. Our guiding research question through the design process was: What design dimensions (or decisions) need to be addressed, to create an animated ASL character system? To answer that question, we formulated design goals and derived design dimensions from those goals, described as follows.

\section{Framework}

We limited the design problem to animating an existing character system, si5s [3], in order to build on substantial prior work and include influences from the Deaf community. We chose si5s for the following reasons:

- It was designed by Deaf community members, and endorsed by Gallaudet University (e.g., [57]), the leading university in the world serving primarily Deaf students.

- It is featural, meaning that it represents sign features separately with component symbols, which allows for movement symbols to be replaced by animations of other symbols (as in Figure 2). For example, where the notation indicates that a hand moves up and down, we remove the motion symbol and animate the referenced handshape symbol instead.

- Its handshape and location symbols are fairly iconic, meaning that they roughly resemble handshapes and body parts, which contributes to understandability without training.
- Elegance and simplicity of the system.

- The symbols comprising the character set are publicly available for download from ASLized [2].

\section{Design Goals}

Our goal was to animate the line-drawn symbols of si5s, so that the animations resemble live sign movements. Based on related work, knowledge of ASL, and our experience with both character systems and animation design, we broke this high-level goal down into specific goals:

1. 3D movement: The animations should represent 3D movements (left/right, up/down, and in/out).

2. Hand orientation: The animations should represent changes in hand orientation, which occur in 3D space.

3. Speed: The speed of the sign movement, which can communicate meaning, should be represented.

4. Start and end: The animations should indicate where movements start and end, including whether the movement is repeated, and if so how many times.

5. Availability: The animations should be available (present on the screen) when the reader wants them.

\section{Design Dimensions}

In order to design effective animations, we broke the design problem down into a series of decisions. To ensure that the hand orientations considered are comprehensive and linguistically sound, they are taken from Stokoe notation [51]. ${ }^{8} \mathrm{We}$ created at least two designs for each dimension. The design dimensions are outlined below, with our designs shown in Table 2.Our design process does not handle non-manuals specially. si5s represents most non-manuals through iconic symbols, which our animated prototype adopts.

1. X-axis movement: Movement left/right relative to the signer.

2. Y-axis movement: Movement up/down relative to the signer.

3. Z-axis movement: Movement in/out of the signer's body.

4. Facing up or down: The directions that the fingers point.

5. Toward or away from signer: Whether the palm faces toward or away from the signer.

6. Toward or away from center: Whether the palm faces toward the center of the body, or away.

7. Palm up or down: Whether the palm faces up or down.

8. Start of movement: In what position the movement starts (which can be ambiguous since animations play on a loop).

9. Repeated movement: How many times a movement is repeated in the sign.

10. Display mode: Which portion of text is animated and displayed, and how to navigate the text.

\footnotetext{
${ }^{8}$ The only hand orientation pair omitted - straight or bent wrist - is not explicitly written in si5s, which does not depict the wrist.
} 
a) Use the drop-down menu to explore 4 ways of viewing the story below. b) How should the animation indicate that a sign movement is beginning? c) Adjust the sliders so each animation speed is appropriate.

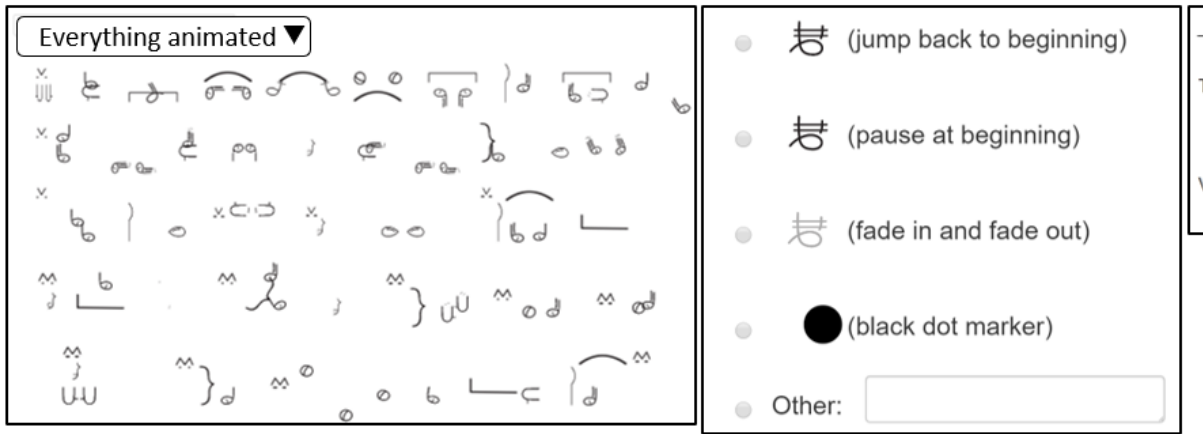

\begin{tabular}{|c|c|c|}
\hline English & ASL & Speed \\
\hline TIRED & 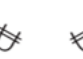 & F目- \\
\hline VERY TIRED & \#\# & \\
\hline
\end{tabular}

Figure 8: Screen shots of the workshop website.

\section{DESIGN PROBE WORKSHOP}

To understand which designs ASL users prefer for each design dimension (described above), and to determine if the system is usable in practice, we ran a design probe workshop with deaf and hard-of-hearing ASL users. We used participatory design principles to involve potential users in the design process.

\section{Participants}

The workshop was run at a university serving primarily Deaf students, with IRB approval. Participants were recruited through relevant email lists, fliers, and word-of-mouth. A total of 15 students participated. Group demographics: Age: 20-41 ( $\mathrm{m}=28)$; Gender: 10 Male, 5 Female; Identity: 13 Deaf, 3 Hard-of-hearing; ASL Experience: all ASL users, 8 from childhood, 7 from young adulthood; Known ASL character systems: 8 SignWriting, 3 English gloss, 1 si5s.

\section{Procedure}

The workshop took place in a university conference room, for 1.5 hours. Interpreters were available throughout. In compensation, participants received snacks, drinks, and a $\$ 20$ Amazon gift card. The basic procedures were:

1. Introductions by everyone present. ( $5 \mathrm{~min}$ )

2. Slide presentation by the researchers explaining the idea of creating an animated ASL character system. (10 min)

3. Participants visited a website to view designs for each design dimension, and input their preferences. (15 $\mathrm{min})$

4. Participants implemented animated characters by hand in groups of 2-3, for a given set of signs. ${ }^{9}(30 \mathrm{~min})$

5. Open discussion. (30 min)

\section{Materials}

Slides The introductory slide presentation consisted of slides explaining the concept of designing an animated character system, and providing description and background on si5s.

Website Participants accessed a website using personal computers (laptops). The website 1) collected basic demographics, 2) showed participants our designs for each design dimension and elicited their preference, and 3) asked for open feedback.

\footnotetext{
${ }^{9}$ While the animated system is not designed for paper, drawings are easily accessible and commonly used in animation design.
}

Participants selected their preferred design for each design dimension through multiple-choice questions, with write-in "other" options (Figure 8a-b). The animated designs were implemented in Javascript/HTML. In-line tables were used to build characters comprising multiple symbols. A long passage [14] was implemented for each display mode (dim 10), with line wrapping (a standard feature of text on computers).

Participants tuned the animation speed for three pairs of signs using a slider (Figure 8c). The signs were: TIRED/VERY TIRED, STROLL/WALK FAST, and HAPPY/VERY HAPPY. These sign pairs were chosen to involve the same movement trajectory, differentiated only by execution speed. The website provided a set of sliders for tuning. When a slider was dragged, the speed of the corresponding animation updated accordingly. The animations were implemented as CSS keyframe animations, with Javascript linking the slider to the display.

Drawing Materials Participants were given printed paper packets, with instructions for drawing character animations for four signs, blank cartoon strips for their drawings, and space to write descriptions and thoughts. They were given pencils, and 3D-printed plastic stencils of si5s symbols at various sizes created by the researchers. The signs were those from the opportunity evaluation study chosen for diversity: WHERE, UNDERSTAND, MAYBE, and MOTIVATION (Figure 9).

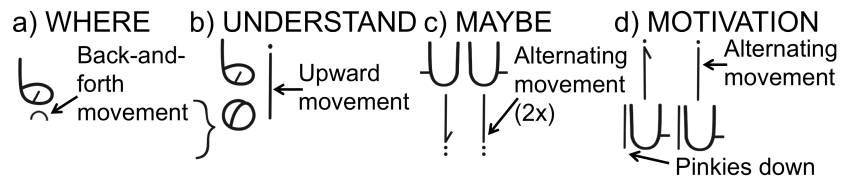

Figure 9: The packet's four signs, in si5s. Participants replaced movement symbols (annotated) with drawn animations.

\section{Results}

The workshop revealed a preference for designs that visually resemble live signing, showed that the system was viable in that people could implement (create content in) it, and highlighted subjective decisions required to create animations.

\section{Design Dimension Preferences}

Participants' selected preferences for the design dimensions are shown in Table 2. For representing 3D movements - movement direction (dim 1-3) and changes in hand orientation (dim 4-7) - participants typically preferred designs that mimic how these aspects of 3D movements are perceived in live signing. For movement direction (dim 1-3), participants preferred 


\begin{tabular}{|c|c|c|c|c|c|c|}
\hline Design & & The Number & of Participants who Prefer & ed Each Design & & \\
\hline 1. X-Axis & horz transl & diag transl, sizing & & & & other \\
\hline Movement & $\emptyset^{+}$ & 丰 & & & & \\
\hline & 11 & 3 & & & & 1 \\
\hline 2. Y-Axis & vert transl & sizing & & & & other \\
\hline Movement & 吾 & 辛 & & & & \\
\hline & 14 & 1 & & & & 0 \\
\hline 3. Z-Axis & sizing & diag transl & diag transl, sizing & vert transl & & other \\
\hline Movement & 吾 & も & 春 & 咅 & & \\
\hline & 12 & 2 & 1 & 0 & & 0 \\
\hline 4 Finoers & $180^{\circ}$ rot & fading & & & & other \\
\hline up/down & 夆年 & E & & & & \\
\hline & 14 & 1 & & & & 0 \\
\hline 5. Toward/ & horz refl & horz refl, fading & vert refl & & & other \\
\hline away signer & あळ & б & б & & & \\
\hline & 10 & 3 & 1 & & & 1 \\
\hline $\begin{array}{l}\text { 6. Toward/ } \\
\text { away center }\end{array}$ & $\begin{array}{l}180^{\circ} \text { rot, stretch } \\
d ?\end{array}$ & $\begin{array}{l}180^{\circ} \text { rot } \\
\text { ottle }\end{array}$ & $\begin{array}{l}180^{\circ} \text { rot, stretch, shadow } \\
d \mathbb{P}\end{array}$ & & & other \\
\hline & 8 & 5 & 1 & & & 1 \\
\hline $\begin{array}{l}\text { 7. Palm } \\
\text { un/down }\end{array}$ & horz refl, stretch & $\begin{array}{l}\text { vert refl } \\
も \supseteqq\end{array}$ & horz refl, stretch, shadow & & & other \\
\hline & 9 & 3 & 0 & & & 3 \\
\hline 8. Start of & $\begin{array}{l}\text { no signal } \\
\text { も }\end{array}$ & fade & $\begin{array}{l}\text { pause } \\
\text { 封 }\end{array}$ & black dot marker & & other \\
\hline & 5 & 4 & 3 & 2 & & 1 \\
\hline 9. Repeated & $\begin{array}{l}\text { arrow, reps written } \\
\sqrt{3 x}\end{array}$ & dots above & inter-set fade & & inter-set pause & other \\
\hline & 芦 & $\theta^{+}$ & 丰 & 帮 & 苨 & \\
\hline & 5 & 5 & 3 & 1 & 1 & 0 \\
\hline $\begin{array}{l}\text { 10. Display } \\
\text { mode }\end{array}$ & $\begin{array}{l}\text { single animated } \\
\text { character dis- } \\
\text { played (left/right } \\
\text { keys to progress) }\end{array}$ & $\begin{array}{l}\text { full text dis- } \\
\text { played, anima- } \\
\text { tion on demand } \\
\text { (on mouse hover) }\end{array}$ & $\begin{array}{l}\text { full animated text dis- } \\
\text { played }\end{array}$ & $\begin{array}{l}\text { full text displayed, } \\
\text { sliding window an- } \\
\text { imated (left/right } \\
\text { keys to progress) }\end{array}$ & & other \\
\hline & 8 & 5 & 2 & 0 & & 0 \\
\hline
\end{tabular}

Table 2: Participants' preferences on designs for each design dimension. (Viewed in Adobe Reader, table contains animations.) Key: diag: diagonal, horz: horizontal, vert: vertical, refl: reflection, rot: rotation, transl: translation, reps=repetitions.

horizontal translation, vertical translation, and size change, respectively (mimicking a hand moving left/right, up/down, and towards/away the face, respectively). For changes in hand orientation (dim 4-7), participants preferred rotations and reflections, resembling a hand rotating or flipping; and horizontal stretching, resembling an angled hand with one part closer (larger) than the rest. Participants largely agreed on these designs, with $>50 \%$ agreement for each dimension.

Participants had a lower level of agreement for how to represent a movement's start, number of repetitions, and display mode (dim 8-10). To represent the start of a movement, the most popular design (at 5/15 participants) was no signal - not using any marker to indicate the movement's start. Because animations play on a loop, in this design the movement start is ambiguous, suggesting that some level of ambiguity in exchange for simplicity is acceptable, as in other writing systems. For representing an $\mathrm{N}$-time repetition, the most popular designs were an arrow with the $N$ written and a set of $N$ dots above the animation (both at 5/15 participants), suggesting that both are viable options. For display mode, most participants $(8 / 15)$ preferred the single sign view, though a significant number preferred the on-demand and full page animated designs, suggesting that giving users a choice of display modes is appropriate. In the future, even more display modes could be offered, e.g., using eye tracking to trigger animations.

\section{Animation Speed Preferences}

Participants systematically and consistently tuned animation speed, suggesting that animation speed is a salient design di- 


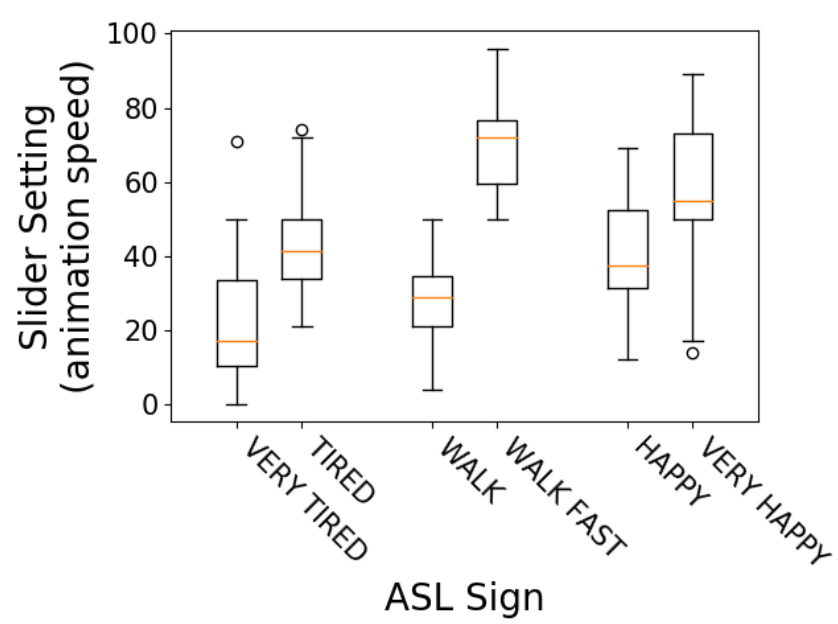

Figure 10: Participant animation speed selections. Slider range (0-100) corresponds to a linear time scale for a single animation repetition (5-0.4s). Higher is faster.

mension, useful for differentiating ASL characters. Figure 10 shows the speeds that participants selected for the animated notation of six signs. Significantly different speeds were selected for pairs of signs that differ only by speed in live signing - TIRED/VERY TIRED $(t(14)=3.02, p=$ $.005)$, WALK/WALK FAST $(t(14)=-8.66, p<<.005)$, HAPPY/VERY HAPPY $(t(14)=2.10, p=.046)$.

The community's consensus around relative speeds suggests that 1) animation speed can affect perceived meaning, and 2 ) the community generally agrees on what speeds are appropriate for various sign characters, suggesting it is possible to create an animated character systems with speeds that the community will largely accept. Furthermore, while speed is difficult to represent in stationary notation (and is not represented in the stationary si5s notation), speed is an inextricable property of animation. This natural portrayal of a semantically meaningful aspect of the language highlights the potential benefit of an animated character system for ASL.

\section{The System in Practice}

All seven groups successfully implemented the system (designed animations to replace si5s movement symbols, e.g., Figure 11) for at least one sign. Six groups drew animations for all four signs. The remaining group only animated WHERE. We believe they were unsure how to draw animations for the more complex signs. Given that participants received only a 5-minute introduction to the character system and had limited drawing time, it is likely that with practice or further instruction, this last group would have completed all signs.

WHERE Participants' drawings for WHERE were very consistent. All seven groups drew the 1-handshape symbol rotating about $20^{\circ}$ clockwise and counterclockwise. One group kept the arch symbol below the handshape symbol, which represented the movement trajectory in the (stationary) si5s notation. One group added two dots to upper-right corner of their frames, annotating their design " 2 dots for repetition." Groups also differed in how many frames they drew.

UNDERSTAND Four groups drew the S-handshape symbol transitioning to the 1-handshape during an upward movement.

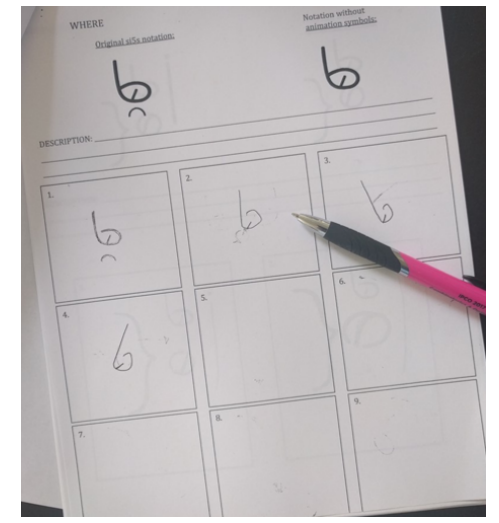

Figure 11: One group's animation design for the sign WHERE.

Of these, one group added facial expressions, drawing flat eyebrows at the beginning and raised ones at the end, indicating that the sign is a question, as in "Understand?". Another of the four groups drew the index finger gradually extending, rather than jumping from one handshape symbol to the next. Two groups made their own notation with arrows pointing upwards.

MAYBE Five groups drew alternating vertical translations of the hand shape symbols, to indicate that the hands move up and down during the sign. One group changed the size of the hand shape symbols to indicate up/down movement, alternately drawing one handshape smaller than the other.

MOTIVATION Six groups again drew alternating vertical translations of the hand shape symbols, to represent the hands rubbing back and forth. Their notation differed from that for MAYBE in the direction of the thumb marks on the handshape notation (corresponding to a horizontal reflection of the handshape symbol). One of these groups rotated the handshape symbols to lie on their side, mimicking the hands with pinkies down during the live sign. Three groups added a line next to the pinkie of the handshape symbol to indicate that the pinkies point down, as in the stationary si5s notation. One group added two dots at the upper-right corner to indicate repetition.

\section{Qualitative Feedback}

Participants generally enjoyed the idea of creating an animated character system, several calling it "fun" and "creative." A few participants questioned the purpose of creating a new writing system, as they were used to using English or ASL video. Several wanted to continue the conversation beyond the end of the workshop, asking questions, discussing the design problems and opportunities, and potential use cases.

During the written portion of the workshop, participants noted difficulties in designing animations. Several noted that it was difficult to represent certain aspects of ASL in the system, for example one participant noting "I think that the illustration of the hands' orientation in some cases could use a new way of expression; one that more clearly communicates the position of the hands." Participants also noted that it is difficult to decide on a single representation for a movement, raising questions such as whether the animation should gradually transition between handshapes or jump from one to the next.

Many students were interested in discussing potential use cases for an animated character system. One participant noted 
that he would like to use the system to help with finding ASL content. For example, he would like to enter a sentence in the animated writing system into a search engine, which would return matching portions of ASL videos. Other participants noted educational benefits of an animated writing system that demonstrates sign movements for people learning ASL, instead of complicated drawings of movements or stationary notation that does not convey movement. Other noted use cases included texting friends and taking notes.

\section{DISCUSSION AND FUTURE WORK}

The animation capabilities of modern screens offer an exciting opportunity to create iconic character systems for sign languages. Existing writing systems have been limited to what is easily handwritten - stationary lines, curves, and dots - which fails to intuitively capture sign movements and consequently can be difficult to learn. An animated writing system represents sign movements directly, precluding the need to memorize complex movement notation. Animation also inherently depicts sign speed, a semantically meaningful aspect of ASL with gradations not easily captured symbolically. An intuitive character system could greatly benefit the Deaf community, whose primary language does not have a standard written form, and who have low literacy rates. Animated character systems could also be useful in other domains involving gesture, e.g., to document surgery procedures or the performing arts.

While we present the first prototype of an animated sign language character system, we do not claim that our proposed system is better to learn and understand. Our pilot study tested only a small set of signs. The set was chosen to span diverse movements, but was far from complete, leaving room for future studies with more comprehensive sets of signs. Our pilot study also evaluated recognizability of isolated characters, whereas longer passages providing context are more common in daily life (though our design probe workshop did present a complete story). The animated notation was more identifiable for individual signs, providing some evidence that animations can increase iconicity and identifiability. Determining conclusively if animation is beneficial requires future work.

We also only explored a single framework for creating an animated character system - taking si5s, an existing stationary writing system, and replacing its movement characters with animation. Starting with an existing character system has the benefit of providing a hand-writable version. It is possible that animating a different character system, or building an animated system from scratch with animation in mind would produce a more favorable result. Creating an animated system from scratch is perhaps the most compelling, especially if we are not interested in preserving handwrite-ability, which becomes less crucial as computers become increasingly pervasive.

There is an interesting trade-off between handwrite-ability and iconicity in sign language character systems. For example, it is possible to create an animated character system that can still be largely written, or has a hand-writable version (e.g., si5s and animated si5s). To provide the benefit of handwriting, such systems must be limited to monochromatic lines, curves, and dots, which limit the extent to which the text resembles a signing human. Alternatively, we can sacrifice the ability to write by hand in favor of creating truly iconic text involving multiple colors, complex shapes, and various animations. Many people currently write by hand, but it is not clear how valuable handwriting will be in the future, as we move away from paper towards electronic resources. Exploring input methods for animated character systems makes rich future work.

Like all writing systems, sign language character systems abstract away some of the live language. For spoken languages like English, the community has agreed on which aspects of the language text must capture, and which may be left out. For a sign language character system to be widely accepted, the community must reach a similar consensus about which parts of the live language must be captured in text. Ambiguity in writing systems is also generally accepted. For example, heteronyms are words that are spelled the same but pronounced differently, e.g., "lead" (the metal) and "lead" (to guide). They are a subcategory of homographs, words with identical spelling but different meanings. Analogously, two signs might share notation but differ in meaning or execution. For a sign language character system to succeed, the community must reach agreement on acceptable ambiguities.

Because we are the first to propose creating an animated sign language character system, this work raises many new research questions. Our main research question was: Is there value in creating an animated ASL character system? This work suggests that the answer is "yes," supporting vast future work: exploring the animated character system design space further, examining potential impacts on literacy and childhood language development, developing teaching methods for animated character systems, exploring use cases for sign language classes, examining gender differences (not statistically significant in our two studies), studying effects of reading long animated passages, developing complementary input methods, integrating animated scripts into existing resources, providing support for a community of character system users and contributers, and analyzing generated corpuses of animated text to spur developments in sign language translation and linguistics.

\section{CONCLUSION}

In this work, we introduce the idea of using animation in a sign language character system, to improve resemblance to live signing and potentially improve learnability. We present an opportunity evaluation study, showing that people find existing character systems difficult to learn, and the potential for animation to improve notation recognizability. We also identify design dimensions for animating an existing character system, and run a design probe workshop with ASL users to shed light on appropriate designs for those dimensions.

As computer scientists continue to develop new interfaces (e.g., AR/VR), the constraints on possible text designs will continue to decrease. Continuing to explore ways to leverage new affordances to improve text displays is a powerful line of research, in particular for users inadequately supported by existing text displays, such as today's sign language users. This work shows the potential of leveraging animation capabilities of modern screens to reimagine sign language text, and we encourage other researchers and developers to similarly leverage modern affordances to improve access. 


\section{ACKNOWLEDGEMENTS}

We thank the Harlan Hahn Endowment Fund for support. This material is also based upon work supported by the National Science Foundation under grant CNS-1539179. Any opinions, findings, and conclusions or recommendations expressed in this material are those of the authors and do not necessarily reflect the views of the National Science Foundation.

\section{REFERENCES}

1. Aisha Shamsuna Ahmed and Daniel Su Kuen Seong. 2006. SignWriting on Mobile Phones for the Deaf. In Proc. of Mobility. ACM.

2. ASLized! 2017. The Official American Sign Language Writing Font by ASLized \& si5s.

http://aslized.org/font-download/. (2017). (Accessed 2018-03-02).

3. RA Augustus, E Ritchie, and S Stecker. 2013. The Official American Sign Language Writing Textbook. ASLized (2013).

4. Jeffrey P Bigham, Daniel S Otero, Jessica N DeWitt, Anna Cavender, and Richard E Ladner. 2008. ASL-STEM Forum: A Bottom-Up Approach to Enabling American Sign Language to Grow in STEM Fields. In ICWSM.

5. Susanne Bødker, Pelle Ehn, Dan Sjögren, and Yngve Sundblad. 2000. Co-operative Design-perspectives on 20 years with 'the Scandinavian IT Design Model'. In Proc. of NordiCHI.

6. Danielle Bragg, Shiri Azenkot, and Adam Tauman Kalai. 2016. Reading and Learning Smartfonts. In Proceedings of the 29th Annual Symposium on User Interface Software and Technology. ACM, 391-402.

7. Danielle Bragg, Shiri Azenkot, Kevin Larson, Ann Bessemans, and Adam Tauman Kalai. 2017. Designing and Evaluating Livefonts. In Proceedings of the 30th Annual ACM Symposium on User Interface Software and Technology. ACM, 481-492.

8. Danielle Bragg, Kyle Rector, and Richard E Ladner. 2015. A user-Powered American Sign Language Dictionary. In Proceedings of the 18th ACM Conference on Computer Supported Cooperative Work \& Social Computing. ACM, 1837-1848.

9. Mary Brennan, Martin D Colville, Lilian K Lawson, and Gerry Hughes. 1984. Words in hand: A structural analysis of the signs of British Sign Language. Edinburgh BSL Research Project.

10. Barbara Brownie. 2014. Transforming Type: New Directions in Kinetic Typography. Bloomsbury Publishing.

11. The Unicode Consortium. 2015. The Unicode Standard, Version 8.0.

http://www. unicode.org/versions/Unicode8.0.0/. (2015).

12. Antonio Carlos da Rocha Costa and Graçaliz Pereira Dimuro. 2003. SignWriting and SWML-Paving the way to Sign Language Processing. Atelier Traitement Automatique des Langues des Signes, TALN 2003 (2003).
13. Karin Danielsson, Amir M Naghsh, Dorina Gumm, and Andrew Warr. 2008. Distributed Participatory Design. In CHI'08 extended abstracts on Human factors in computing systems. ACM, 3953-3956.

14. Joseph Davis and Elsie Stecker. 2011. Deaf-Blind Ninja. ASLized! Journal of American Sign Language and Literature. (2011).

15. Matjaž Debevc, Primož Kosec, and Andreas Holzinger. 2011. Improving Multimodal Web Accessibility for Deaf People: Sign Language Interpreter Module. Multimedia Tools and Applications 54, 1 (2011), 181-199.

16. Eleni Efthimiou and Stavroula-Evita Fotinea. 2007. An environment for deaf accessibility to educational content. Greek national project DIANOEMA,(GSRT, M3. 3, id 35), Hammamet-Tunisia, ICTA (2007).

17. Ralph Elliott, JRW Glauert, Vince Jennings, and JR Kennaway. 2004. An Overview of the SiGML Notation and SiGMLSigning Software System. In Sign Language Processing Satellite Workshop of the Fourth International Conference on Language Resources and Evaluation, LREC 2004. 98-104.

18. Christiane Floyd, Wolf-Michael Mehl, Fanny-Michaela Reisin, Gerhard Schmidt, and Gregor Wolf. 1989. Out of Scandinavia: Alternative Approaches to Software Design and System Development. Human-Computer Interaction 4, 4 (1989), 253-350.

19. Kenneth I Forster. 1970. Visual Perception of Rapidly Presented Word Sequences of Varying Complexity. Perception \& Psychophysics 8, 4 (1970), 215-221.

20. Christopher Frauenberger, Judith Good, and Wendy Keay-Bright. 2011. Designing Technology for Children with Special Needs: Bridging Perspectives through Participatory Design. CoDesign 7, 1 (2011), 1-28.

21. Dafydd Gibbon, Ulrike Gut, Benjamin Hell, Karin Looks, Alexandra Thies, and Thorsten Trippel. 2003. A Computational Model of Arm Gestures in Conversation.. In INTERSPEECH.

22. Donald A Grushkin. 2017. Writing Signed Languages: What For? What Form? American Annals of the Deaf 161, 5 (2017), 509-527.

23. Dorina C Gumm, Monique Janneck, and Matthias Finck. 2006. Distributed Participatory Design-a Case Study. In Proc. of the DPD Workshop at NordiCHI, Vol. 2.

24. Thomas Hanke. 2002. iLex-A tool for Sign Language Lexicography and Corpus Analysis. In LREC.

25. Thomas Hanke. 2004. HamNoSys-Representing Sign Language Data in Language Resources and Language Processing Contexts. In LREC, Vol. 4.

26. Frank G Hofmann and Günter Hommel. 1996. Analyzing human gestural motions using acceleration sensors. In Proc. of Gesture Workshop on Progress in Gestural Interaction. Springer-Verlag, 39-59. 
27. Judith A Holt. 1993. Stanford Achievement Test-8th edition: Reading Comprehension Subgroup Results. American Annals of the Deaf 138, 2 (1993), 172-175.

28. Matt Huenerfauth. 2004. A Multi-Path Architecture for Machine Translation of English Text into American Sign Language Animation. In Proc. of the Student Research Workshop at HLT-NAACL. Association for Computational Linguistics, 25-30.

29. Matt Huenerfauth. 2006. Generating American Sign Language Classifier Predicates for English-to-ASL Machine Translation. Ph.D. Dissertation. University of Pennsylvania.

30. Matt Huenerfauth. 2009. A Linguistically Motivated Model for Speed and Pausing in Animations of American Sign Language. ACM Transactions on Accessible Computing (TACCESS) 2, 2 (2009), 9.

31. Matt Huenerfauth and Vicki L Hanson. 2009. Sign Language in the Interface. In The Universal Access Handbook. CRC Press, 1-18.

32. Adam Kendon. 1988. Sign Languages of Aboriginal Australia: Cultural, Semiotic and Communicative Perspectives. Cambridge University Press.

33. Finn Kensing and Jeanette Blomberg. 1998. Participatory Design: Issues and Concerns. Computer Supported Cooperative Work (CSCW) 7, 3-4 (1998), 167-185.

34. Stefan Kopp, Timo Sowa, and Ipke Wachsmuth. 2003. Imitation games with an artificial agent: From mimicking to understanding shape-related iconic gestures. In International Gesture Workshop. Springer, 436-447.

35. Stephen Lindsay, Katie Brittain, Daniel Jackson, Cassim Ladha, Karim Ladha, and Patrick Olivier. 2012. Empathy, participatory design and people with dementia. In Proceedings of the SIGCHI Conference on Human Factors in Computing Systems. ACM, 521-530.

36. Craig H Martell. 2002. An Extensible, Kinematically-Based Gesture Annotation Scheme. Calhoun: The NPS Institutional Archive (2002).

37. Marina McIntire, Don Newkirk, Sandra Hutchins, and Howard Poizner. 1987. Hands and faces: A Preliminary Inventory for Written ASL. Sign Language Studies 56, 1 (1987), 197-241.

38. RDL Meddick and MJ Griffin. 1976. The effect of two-axis vibration on the legibility of reading material. Ergonomics 19, 1 (1976), 21-33.

39. Modern Language Association (MLA). 2018. Enrollments in Languages Other than English in United States Institutions of Higher Education, Summer 2016 and Fall 2016: Preliminary Report.

http://www.mla.org/content/download/83540/2197676/ 2016-Enrollments-Short-Report .pdf. (2018). (Accessed: 2018-03-04).

40. Sara Morrissey and Andy Way. 2005. An Example-Based Approach to Translating Sign Language. Workshop in Example-Based Machine Translation (MT Summit X) (2005), 109-116.
41. Don Newkirk. 1987. SignFont Handbook. San Diego: Emerson and Associates.

42. World Federation of the Deaf (WFD). 2018. Sign Language. https://wfdeaf.org/human-rights/crpd/sign-language/. (2018). (Accessed: 2018-02-20).

43. David Peterson. 2013. Sign Language International Phonetic Alphabet (SLIPA). http://dedalvs. conlang.org/slipa.html. (2013). (Accessed 2017-05-07).

44. Helen Petrie, Wendy Fisher, Kurt Weimann, and Gerhard Weber. 2004. Augmenting Icons for Deaf Computer Users. In CHI'04 Extended Abstracts on Human Factors in Computing Systems. ACM, 1131-1134.

45. Yair Rand. 2014. SignWriting Keyboard. http://swkb-35431. firebaseapp . com/. (2014). (Accessed 2018-03-02).

46. Interagency Language Roundtable. 1985. Interagency Language Roundtable (ILR) Scale. http://www .govtilr.org/Skills/ILRscale1.htm. (1985). (Accessed: 2018-01-31).

47. Gary S Rubin and Kathleen Turano. 1992. Reading Without Saccadic Eye Movements. Vision research 32, 5 (1992), 895-902.

48. Signing Savvy. 2018. ASL Sign Language Video Dictionary. http://www . signingsavvy. com. (2018). (Accessed 2018-04-05).

49. Douglas Schuler and Aki Namioka. 1993. Participatory Design: Principles and Practices. CRC Press.

50. William C Stokoe. 1960. Sign Language Structure: An Outline of the Visual Communication Systems of the American Deaf. Studies in Linguistics: Occasional Papers 8 (1960).

51. William C Stokoe, Dorothy C Casterline, and Carl G Croneberg. 1965 (reissued 1976). A Dictionary of American Sign Language on Linguistic Principles. Linstok Press Silver Spring.

52. Thomas Stone. 2009. ASLSJ. https://sites.google.com/site/aslsignjotting/. (2009). (Accessed 2017-04-05).

53. Katja Straetz, Andreas Kaibel, Vivian Raithel, Marcus Specht, Klaudia Grote, and Florian Kramer. 2004. An E-Learning Environment for Deaf Adults. In Proc. 8th ERCIM workshop.

54. Samuel Supalla. 2012. ASL-phabet. http://www. aslphabet. com/home.html. (2012). (Accessed 2017-05-07).

55. Valerie Sutton. 1995. Lessons in SignWriting: Textbook. SignWriting. 
56. TEDx Talks. 2012. Robert Arnold Augustus at TEDxIslay.

https: //www . youtube . com/watch?v=SYHs3D6jJ_o\&t=271s. (2012). (Accessed 2017-05-04).

57. Gallaudet University. 2017-2018. PST 275 - Introduction to ASL Writing: Si5s System.

http://www.gallaudet.edu/academic-catalog/ center-for-continuing-studies/pst-courses. (2017-2018). (Accessed 2018-03-02).

58. Clayton Valli and Ceil Lucas. 2000. Linguistics of American Sign Language: An Introduction. Gallaudet University Press.

59. Hua Wang, Helmut Prendinger, and Takeo Igarashi. 2004. Communicating Emotions in Online Chat using Physiological Sensors and Animated Text. In CHI'O4 extended abstracts on Human factors in computing systems. ACM, 1171-1174.
60. Christopher Richard Wren, Ali Azarbayejani, Trevor Darrell, and Alex Paul Pentland. 1997. Pfinder: Real-Time Tracking of the Human Body. IEEE Transactions on Pattern Analysis and Machine Intelligence 19, 7 (1997), 780-785.

61. Norziha Megat Mohd Zainuddin, Halimah Badioze Zaman, and Azlina Ahmad. 2010. A Participatory Design in Developing Prototype an Augmented Reality Book for Deaf Students. In 2nd International Conference on Computer Research and Development. IEEE.

62. Liwei Zhao, Karin Kipper, William Schuler, Christian Vogler, Norman Badler, and Martha Palmer. 2000. A Machine Translation System from English to American Sign Language. Envisioning Machine Translation in the Information Future (2000), 191-193. 